\title{
Errata
}

\section{Case report 357}

(Skeletal Radiology, Volume 15, Number 3, 1986, pp. 242-246)

Please note the following corrections to the above mentioned case report:

\section{On page 242 and 243, the captions of Figs. 1-3 should read:}

Figs. 1. A An anteroposterior view of the lumbar spine obtained in April, 1983 shows destruction of the body and pedicles of L4. An increase in density is present in the vertebral body, possibly from previous radiation therapy which was given early in 1982 after a laminectomy was performed. B A lateral lumbar myelogram obtained in April 1983 shows marked constriction and compression of the thecal sac by an extradural soft tissue mass, presumably associated with the destructive lesion of the vertebral body of L4. The opacified spinal canal is deviated posteriorly and sharply indented on its anterior surface over a long expanse. C A computed tomogram over L 4 demonstrates vividly the destruction of this vertebral body and its pedicles. Observe the posteriorly-displaced and compressed thecal sac by the mass associated with the destroyed vertebra

Fig. 2. An anteroposterior film of the lumbar spine obtained in April 1982 (1 year before the admission of the patient to the Orthopaedic Institute) shows poor definition of both pedicles of L 4 - a relatively subtle finding. These were the only roentgenograms that could be recovered prior to admission of the patient to the Orthopaedic Institute in April 1984

Fig. 3. A A lateral film of the thoracic spine obtained in April 1984 shows considerable compression and wedging of the vertebral body of T6. B A radionuclide scan obtained on the same date shows increased uptake of the isotope on the left side of the vertebra of T6 as well as the posterior segments of the upper ribs. C A lateral thoracic myelogram obtained ion April 1984 shows extradural compression of the thecal sac by a soft tissue mass opposite T 5 , T 6 and $\mathrm{T} 7$, with an almost complete extradural block opposite T6. D A computed tomogram of T6 (also in April 1984) demonstrates the intraspinal extension of the tumor compressing the right side of the thecal sac

On page 244, 2nd column, the 4th paragraph should read: Immunohistochemical studies performed in this case showed that the cells of the chordoma reacted positively to antibodies against S 100 protein and cytokeratin, as indicated in the literature [9-12]. Chondrosarcoma is a neoplasm that histologically may resemble chordoma; this lesion too, is known to contain $\mathrm{S} 100$ protein $[9,12]$. However, the cells in chondrosarcoma react negatively to the antibodies against cytokeratin. Therefore, cytokeratin is considered to be a useful immunohistochemical marker in the histological distinction between chordoma and chondrosarcoma [12]. Furthermore, the presence of cytokeratin in chordoma suggests that this neoplasm, arising from the rem-

\section{Announcement*}

\section{Sixth Annual Masters Diagnostic Radiology Conference \\ February 22-27, 1987 \\ Maui, Hawaii}

The meeting will emphasize lectures in skeletal, chest and interventional radiology as well as CT, MRI, ultrasound and nuclear medicine.

For further information contact: Maurice M. Reeder, nants of the notochordal tissue, is of epithelial origin [10].

On page 246, 1st column, the last paragraph should read: In summary a fascinating case is presented in a 54-year-old man who developed a chordoma of the fourth lumbar vertebra which was treated by radiotherapy, with good results. The man remained asymptomatic relatively for 15 months and then presented with recurrence of back pain and neurological deficits. Plain films, CT and myelography showed considerable destruction of the body of $L 4$ with a sclerotic pattern suggesting the effects of previous radiotherapy.
M.D., Program Director, Chairman, Department of Radiology, University of Hawaii School of Medicine, 1356 Lusitana Street, Room 502, Honolulu, Hawaii 96813, USA, telephone (808) $531-6471$

* Announcements for continuing medical education opportunities are limited to one paragraph containing the title of the meeting, sponsor, place, dates, and name and address of contact person. A brief statement regarding meeting emphasis may be included. Lists of faculty, free-time tours, registration fees and other elaborations will not be accepted. 